\title{
A Novel Level Set Method for Ultrasonic Cardiogram Segmentation Based On Chan-Vese Model
}

\author{
Yuzhou Yang, Hong Yan, Yuhua Yao, Xuemin Yin, Meng Xiao, Jinzhong Song \\ China Astronaut Research and Training Center, Beijing 100094, China \\ dove_yyz@126.com, hholden@sina.com, xiaomeng16@126.com, sjinzhongkx@163.com
}

\begin{abstract}
Level Set Method based on Chan-Vese(C-V) model is widely used in image processing and computer vision. However, there are some drawbacks when $\mathrm{C}-\mathrm{V}$ model processes Ultrasonic Cardiogram(UCG) images. For example, the accuracy is influenced by noise and speckle in UCG image and some problems such as numerical error and time consuming are caused by re-initialization in level set evolution. Therefore, a novel level set method based on $\mathrm{C}-\mathrm{V}$ model was proposed in this paper. First of all, the C-V's Partial Differential Equation(PDE) was improved. Second, three signed distance penalizing energy function was analyzed and compared, then the best one which forces the level set function(LSF) to be close to a signed distance function was chosen in this paper. Experiments results showed that the proposed method not only eliminated the effect of speckles on UCG image segmentation, but also reduced the computational cost and avoided numerical errors caused by reinitialization. Besides, the obtained contour curve was much smoother.

Index Terms - Ultrasonic Cardiogram (UCG)image segmentation, level set method, Chan-Vese $(\mathrm{C}-\mathrm{V})$ model, re-initialization
\end{abstract}

\section{Introduction}

As an indispensable inspecting technique for cardiac disease, Ultrasonic Cardiogram(UCG) has several main advantages such as non-invasive, low-price and Real-time. However, because of the high noise, speckle, wide fuzzy boundary region and man-made boundary in UCG, the quality of UCG image is very poor. Especially speckle noise, which makes gray gradient(boundary) occur everywhere in the internal UCG image. Hence, it is more difficult to extract the boundary of UCG than X-CT and MRI image.

In the past two decades, active contour models (ACMs)[1] have been widely used in image processing and computer vision, especially for image segmentation. The original ACM was proposed by Kass et al [1]. An energy function, which can restrict close curve, was defined[1]. Then the curve approached the desired boundary by minimizing the energy function. Problems associated with initialization and poor ability to handling topological changes, however, had limited its utility. To eliminate the above drawbacks, Geometric ACM, also called level set method, which implicitly represents the curve by zero level of a high dimensional function. was proposed later by Osher and Sethian [2].

Only the local boundary information can be used by the traditional level set method in image segmentation, and it is difficult to acquire desired result when there are fuzzy or discrete boundary region. Therefore, Mumford and Shah[3] proposed the level set method based on Mumford-Shah(M-S) model which used the global information in the homogeneous region. Later, Chan-Vese(C-V) model[4] was proposed by simplifying the M-S model. The $\mathrm{C}-\mathrm{V}$ model has a perfect utility when disposing fuzzy and discrete boundary which occur in UCG image. However, the C-V model also stops the curve on the speckle boundary but it is not expected. Besides, although re-initialization has been extensively implemented to keep the evolving level set function close to a signed distance function, it may incorrectly move the zero level set away from the expected position[5].

$\mathrm{C}-\mathrm{V}$ model was improved in this paper to solve the above problems on segmenting UCG image. First, the C-V's Partial Differential Equation(PDE) was improved by replacing $\delta(x)$ with $q(x)$. Second, we analyzed and compared three signed distance penalizing energy function which forces the level set function to be close to a signed distance function, then chose the best one to add to the C-V's PDE. Therefore our method completely avoided the costly re-initialization procedure. Experimental results showed that our method had an outstanding effect on UCG image segmentation.

\section{Background}

A. Level Set Method

As one kind of Active contour models, level set method is formulated in terms of a dynamic parametric contour $C(s, t):[0,1] \times[0, \infty] \rightarrow R^{2}$ with a spatial parameter $s$ and a temporal variable $t$. The curve evolution can be expressed as

$$
\frac{\partial C(s, t)}{\partial t}=F \mathrm{~N}
$$

where $F$ is the speed function[2] which controls the motion of the contour, and $\mathrm{N}$ respect the inward normal vector to the curve $\mathrm{C}$.

Level set method represents the dynamic contour $C(s, t)$ as the zero level set $C(t)=\{(x, y) \mid \phi(t, x, y)=0\}$ of a time dependent level set function(LSF) $\phi(t, x, y)$. The LSF $\phi(t, x, y)$ is represented by the signed distance function(SDF) which takes positive values inside the zero level contour and negative values outside. The inward normal vector can be expressed as $\mathrm{N}=-\nabla \phi / \nabla \phi$, where $\nabla$ is the gradient operator. Then, the curve evolution equation (1) is converted to the following (PDE) formula

$$
\frac{\partial \phi}{\partial t}=F|\nabla \phi|
$$




\section{B. Drawbacks Associated with Re-initialization}

The initial LSF $\phi$ was proposed as $\phi(x)=1 \pm d i s t^{2}(x)$ [2], where $\operatorname{dist}(\cdot)$ is a distance function and " \pm " denotes whether the region is inside or outside the contour. However, when LSF evolution is proceeding, it is of big importance to reinitialize $\phi$ to the SDF[4][5], which prevents LSF from being too steep or too flat to accurately calculate. A standard re-initialization method is to solve the following evolution equation:

$$
\left\{\begin{array}{c}
\psi_{T}=\operatorname{sign}(\phi(t))(1-|\nabla \psi|) \\
\psi(0, \cdot)=\phi(t, \cdot)
\end{array}\right.
$$

where $\phi(t, \cdot)$ is the result $\phi$ at time $t$. Then $\psi$ will be the new $\phi(t, \cdot)$ Although re-initialization has been extensively used as a numerical remedy for maintaining stable curve evolution and desirable results, it may not only incorrectly move the zero level set away from the expected position, but also the process can be complicated and expensive.[5] Thus it is necessary to develop a method to avoid re-initialization.

\section{C-V model}

The purpose of image segmentation is to separate the regions from different parts which have the homogeneity gray. Every region can be expressed by its boundary. The local and gradient information of image are widely used as image driving force by general geometric ACMs such as Geodesic Active Contour model[7].

A level set model, which was proposed by Chan and Vese[4] based on the simplified M-S model, used the global information of image. The image $I(x, y)$ with the domain $\Omega$ is divided by closed boundary $\mathrm{C}$ into two homogeneous regions: the target region $\omega_{a}$ (inside C) and the background region $\omega_{b}$ (outside C), $c_{1}$ and $c_{2}$ are the mean gray in region $\omega_{a}$ and $\omega_{b}$.

The speed function, which is $F$ in formula (1), also called as the energy function, is expressed as:

$$
\begin{aligned}
& F\left(c_{1}, c_{2}, C\right)=\mu L(C)+v S(C)+\lambda_{1} \int_{\text {inside }(C)}\left|I(x, y)-c_{1}\right|^{2} d x d y \\
& +\lambda_{1} \int_{\text {outside }(C)}\left|I(x, y)-c_{2}\right|^{2} d x d y
\end{aligned}
$$

where $L(C)$ is the length of the closed boundary $C, S(C)$ is the area of the region inside $C$, and $\mu, v \geq 0, \lambda_{1}, \lambda_{2}>0$ are fixed parameters. By taking place $F$ with $F\left(c_{1}, c_{2}, C\right)$, the curve evolution equation (2) is changed as

$$
\frac{\partial \phi}{\partial t}=F\left(c_{1}, c_{2}, C\right)|\nabla \phi|
$$

$F(C)$ reaches the minimum when the curve $C$ is the boundary of the two homogeneous regions. Therefore, the final boundary $C$ can be obtained by minimizing formula (4), which is expressed as

$$
\inf _{c_{1}, c_{2}, C} F\left(c_{1}, c_{2}, C\right)
$$

As global information of image was used, the global optimal segmentation results can be obtained by optimizing formula(6).

$\phi_{0}$ is assumed as the initial LSF based on initial curve $C_{0}$, that is $\left\{C_{0} \mid \phi_{0}(x, y)=0\right\}$, let $\phi$ be the SDF which takes positive values inside the zero level contour and negative values outside, namely $\phi($ inside $(C))>0, \phi($ outside $(C))<0$. The PDE expressed by LSF $\phi$ was deduced by using the Euler Lagrange method, and they are shown as follows:

$$
\left\{\begin{array}{c}
c_{1}(\phi)=\frac{\int_{\Omega} I(x, y) H(\phi) d x d y}{\int_{\Omega} H(\phi) d x d y}, c_{2}(\phi)=\frac{\int_{\Omega} I(x, y)(1-H(\phi)) d x d y}{\int_{\Omega}(1-H(\phi)) d x d y} \\
\frac{\partial \phi}{\partial t}=\delta(\phi)\left[\mu \nabla \cdot \frac{\nabla \phi}{|\nabla \phi|}-v-\lambda_{1}\left(I(x, y)-c_{1}\right)^{2}+\lambda_{2}\left(I(x, y)-c_{2}\right)^{2}\right] \\
\phi(0, x, y)=\phi_{0}(x, y)
\end{array}\right.
$$

where $\Omega$ is the domain of the image and LSF, $H(z)$ is Heaviside function, that is $H(z)=\left\{\begin{array}{l}1, z>0 \\ 0, z<0\end{array}, \delta(x)\right.$ is the Dirac function. $H(z)$ was changed by Chan and Vese[4] as

$$
H(z)=\frac{1}{2}\left(1+\frac{2}{\pi} \arctan \left(\frac{z}{\varepsilon}\right)\right)
$$

and $\delta(x)$ is the derivative of $H(z)$, that is

$$
\delta(x)=\frac{\varepsilon}{\pi\left(\varepsilon^{2}+x^{2}\right)}
$$

which is widely used in LSMs[4-6][8-10].

By using the global information of image shown from the formula (7), C-V model has several advantages comparing to the other ACMs[1][3][11] in UCG image segmentation. All the inside and outside edges of the targets with internal empty can be detected by only one initial contour and the initial contour can escape from the constraint of position and shape in the image. Moreover, the model has a significant effect on fuzzy and discrete boundary .

\section{Improved C-V model}

\section{A. Improvement One}

As mentioned previously, the speckle noise in UCG image can be also segmented. However, our main goal is just to segment the ventricle and atrium without speckle. The Dirac function $\delta(\phi)$ in formula(7) limits the use of global information because of the narrow definition[12]. As plotted in Fig. 1(a), $\delta(\phi)$ is close to one only when $\phi(x, y)$ is close to zero, and is a positive value close to zero for other values of $\phi(x, y)$. the result of using $\delta(\phi)$ is that heterogeneous region boundary, which is speckle in the internal empty region, can be detected. For not segmenting the speckle, we replace $\delta(\phi)$ with quadratic function, that is $q(\phi)=a \phi^{2}+1$, and the 
PDE in (7) is changed as

$$
\frac{\partial \phi}{\partial t}=q(\phi)\left[\mu \nabla \cdot \frac{\nabla \phi}{|\nabla \phi|}-v-\lambda_{1}\left(I(x, y)-c_{1}\right)^{2}+\lambda_{2}\left(I(x, y)-c_{2}\right)^{2}\right]
$$

As plotted in Fig. 1(b), $q(\phi)$ is a value close to one for all the values of $\phi(x, y)$ in the image domain so that the SDFs are the same sign in the speckle and internal empty region. Then, the speckle cannot be segmented.

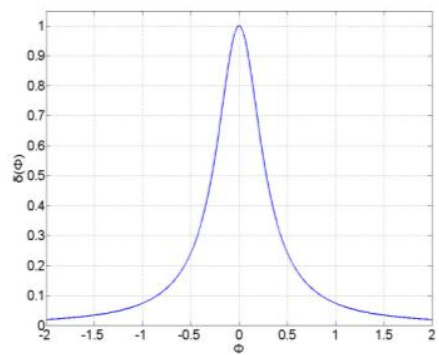

(a)

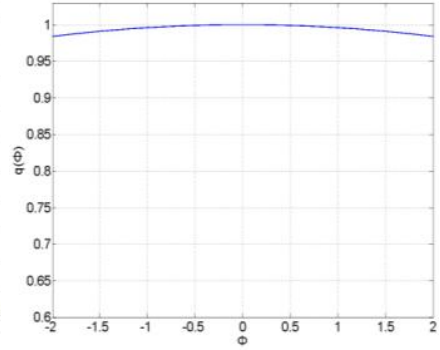

(b)
Fig. 1 (a) the Dirac function $\delta(\phi)$ in formula(9), (b)the quadratic function $q(\phi)$ proposed in this paper

\section{B. Improvement Two}

As discussed in Background section, many problems occur during curve evolution because of re-initialization. In recent years, many methods were proposed aiming to avoid reinitialization. Some researchers[5],[6],[13] proposed a signed distance penalizing energy function $P(\phi)$, of which the purpose is to measure the closeness between an LSF $\phi$ and an $\mathrm{SDF}$ in the domain $\Omega \subset R^{n}, n=2 o r 3$. In this paper, $P(\phi)$ was added to the $\mathrm{C}-\mathrm{V}$ 's curve evolution equation, so the formula (5)is changed as

$$
\frac{\partial \phi}{\partial t}=P(\phi)+F\left(c_{1}, c_{2}, C\right)|\nabla \phi|
$$

$P(\phi)$ was explicitly expressed by Li et al [5]as

$$
P(\phi)=\frac{1}{2} \int_{\Omega}(|\nabla \phi|-1)^{2} d x
$$

and the Gateaux derivative of $P(\phi)$ in (12) is

$$
\frac{\partial P}{\partial \phi}=-\operatorname{div}\left(d_{p}(|\nabla \phi|) \nabla \phi\right)
$$

which is a diffusion equation with rate

$$
d_{p 1}(|\nabla \phi|)=1-(1 /|\nabla \phi|)
$$

Li et al [6] improved $P(\phi)$ of which the diffusion equation rate $d_{p}(|\nabla \phi|)$ is replaced with

$$
d_{p 2}(|\nabla \phi|)=\left\{\begin{array}{c}
\frac{\sin (2 \pi|\nabla \phi|)}{2 \pi|\nabla \phi|}, \text { if }|\nabla \phi| \leq 1 \\
1-\frac{1}{|\nabla \phi|}, \text { if }|\nabla \phi| \geq 1
\end{array}\right.
$$

a constrained level set diffusion rate was proposed in reference [8]as

$$
d_{p 3}(|\nabla \phi|)=\frac{1}{2}\left[1+\frac{2}{\pi}\left(\arctan \left(\frac{|\nabla \phi|-1}{\rho}\right)\right)\right]
$$

where $\rho$ is a fixed parameter.

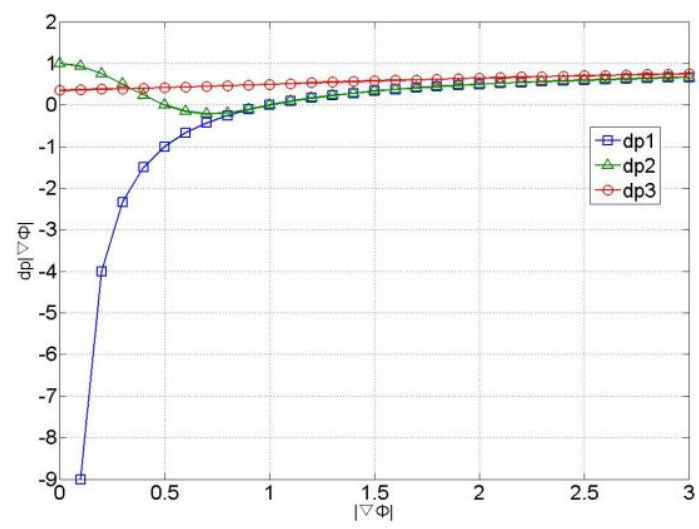

Fig. 2 different diffusion rates

The three diffusion rates are showed in the Fig. 2. We see that for $d_{p 1}(|\nabla \phi|)$, when $|\nabla \phi|>1$, the diffusion rate $d_{p 1}(|\nabla \phi|)$ is positive and the diffusion is forward, which decreases $|\nabla \phi|$. When $|\nabla \phi|<1$ the diffusion rate $d_{p 1}(|\nabla \phi|)$ is negative and the diffusion is backward, which increases $|\nabla \phi|$. However, when $|\nabla \phi|$ is close to zero, the diffusion goes to negative infinity, which may cause the contour disappeared in the image. For $d_{p 3}(|\nabla \phi|)$, the diffusion rate changes smoothly and is positive all the time, which cannot increase $|\nabla \phi|$. For $d_{p 2}(|\nabla \phi|)$, when $|\nabla \phi|<1 / 2$ or $|\nabla \phi|>1$, the diffusion rate is positive and the diffusion is forward, which decreases $|\nabla \phi|$. When $1 / 2<|\nabla \phi|<1$, the diffusion rate is negative, and the diffusion is backward, which increases $|\nabla \phi|$. As mentioned in reference [6], the $d_{p 2}(|\nabla \phi|)$ also has a strong effect on smoothing curve.

Therefore, $d_{p 2}(|\nabla \phi|)$ was chose as the signed distance penalizing energy function through the above analysis and comparison. So the formula (10)Turns into

$$
\begin{aligned}
& \frac{\partial \phi}{\partial t}=\mu \operatorname{div}\left(d_{p 3}(|\nabla \phi| \nabla \phi)\right) \\
& +q(\phi)\left[\mu \nabla \cdot \frac{\nabla \phi}{|\nabla \phi|}-v-\lambda_{1}\left(I(x, y)-c_{1}\right)^{2}+\lambda_{2}\left(I(x, y)-c_{2}\right)^{2}\right]
\end{aligned}
$$




\section{IV . Experiment Results}

The data of this paper is three cardiac cycle UCG from a healthy person including 78 images collected by GE Vivid E9, The size of the image is $434 \times 636$ pixels. In the experiment, the traditional $\mathrm{C}-\mathrm{V}$ model, Li's [6]method and our method were compared. One of the 78 UCG images was chosen as the example to describe the effect of our method. we set $\lambda_{1}=\lambda_{2}=1, v=0.001 \times 255 \times 255$ and $\Delta t=1, \mu=0, \mathrm{a}=-0.004, \varepsilon=1$ for our method. The experiment results are illustrated in the Fig 3 .

Fig3.(b) shows that $\mathrm{C}-\mathrm{V}$ model[4] can detect the interior contour of heart. However, the speckle can be also segmented so that the results affects the accuracy of segmentation. Fig3.(c) shows the result which uses Li's method[6] to segment the UCG image. Obviously, the segmentation cannot finish successfully, and the reason is that the Li's model uses gradient information to evolute contour curve. Not only the interior contour of heart can be accurately segmented, but the speckle can be filtered by using our improved model. Besides, the contour curve is much smoother, as shown in Fig3.(d).

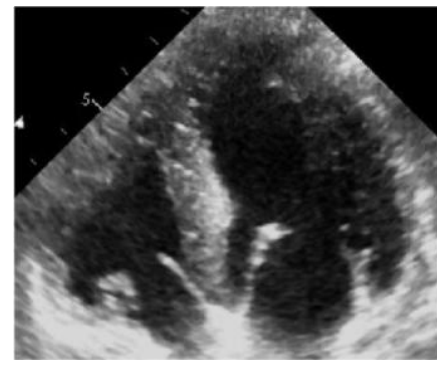

(a)

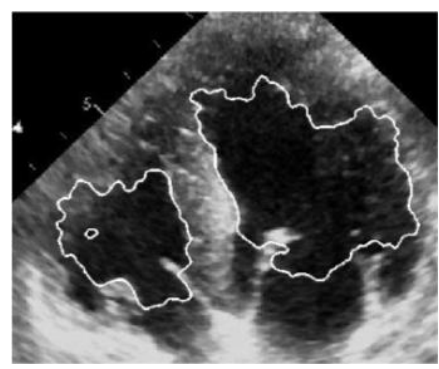

(c)

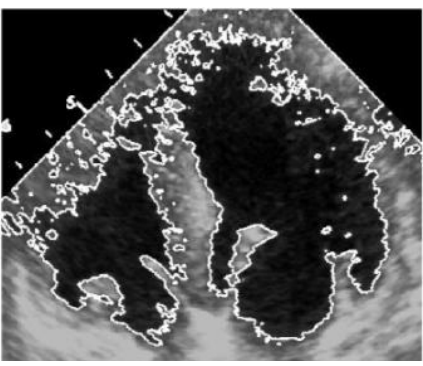

(b)

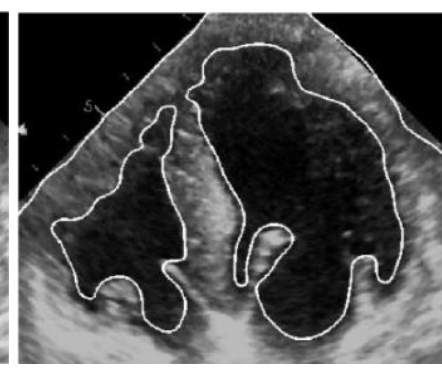

(d)
Fig. 3 (a) the original UCG image, (b)the segmentation result of $\mathrm{C}-\mathrm{V}$ model, (c) the segmentation result of Li's method, (d) the segmentation result of our method

\section{V . Discussion and Conclusion}

In the experiment, our method was compared with Li's method and C-V model. Because of the big noise and considerable number of speckles, there are gradients everywhere in UCG image. Li's method with gradient information as the curve driving force cannot segment the heart contour successfully. It is global information that is used in our method. Global information uses every gray in image and the curve driving force cannot be limited by boundary gradient, therefore our method has a significant effect on the fuzzy and discrete boundary caused by noise and speckle in UCG image. Besides, the initial contour in Li's method must be inside or outside the boundary, but the initial contour in our method can be everywhere in image. Therefore, our method meets the need of auto-segmentation without initializing contour by handwork. By taking place $\delta(x)$ with $q(x)$, our method has a better effect on filtering speckle comparing to C$\mathrm{V}$ model. Obviously, the contour of our method is much smoother because we add a signed distance penalizing energy function $P(\phi)$ which not only avoids re-initialization, but also has a function of smoothing curve.

In summary, the proposed method in this paper is appropriate for UCG image segmentation.

\section{Acknowledgment}

We gratefully acknowledge support from Advanced Space Medico-Engineering Research Project of China(2011SY540 7019,2012SY54B0601,SJ201006), State Key Laboratory of Space Medicine Fundamentals and Application, China Astronaut Research and Training Center(SMFA12B09)

\section{References}

[1] M. Kass, A. Witkin, and D.Terzopoulos, Snakes: Active contour models, International. Journal. Computer. Vison, 1988, 1(4):321-332

[2] S. Osher, J A. Sethian. Fronts propagating with curvature-dependent speed: Algorithms based on Hamilton-Jacobi formulation. Journal of Computational Physics, 1988, 79(1): 12-49.

[3] D. Mumford, J Shah. Optimal approximation by piece-wise smooth functions and associated variational problems. Communication on Pure and Applied Mathematics, 1989,42(5):577-685

[4] F T. Chan, L. Vese. Active contours without edges. IEEE Trans Image Processing, 2001, 10(2):266-277.

[5] C. Li, C. Xu, C. Gui and M. D. Fox, Level set evolution without reinitialization: A new variational formulation, in Proc. IEEE Conf. Comput. Vis. Pattern Recognit. 2005, vol. 1, pp. 430-436.

[6] C. Li, C. Xu, C. Gui and M. D. Fox, Distance regularized level set evolution and its application to image segmentation, IEE Transaction On Image Processing, Vol. 19 pp 3243- 3254

[7] V. Caselles, R. Kimmel, and G. Sapiro, Geodesic active contours, Int. J. Comput. Vis., vol. 22, no. 1, pp. 61-79, Feb. 1997.

[8] X. Xie, "Active Contouring Based on Gradient Vector Interaction and Constrained Level Set Diffusion," IEEE Trans. Image Processing, vol. 19, no. 1, pp. 154-164, Jan. 2010.

[9] C. Li, C. Kao, J. Gore, and Z. Ding, "Implicit Active Contours Driven by Local Binary Fitting Energy," Proc. IEEE Conf. Computer Vision and Pattern Recognition, pp. 1-7, 2007.

[10] K. Zhang, H. Song, and L. Zhang, "Active contours driven by local image fitting energy," Pattern recognition, vol. 43, no. 4, pp. 1199-1206, 2010.

[11] C. Xu and J. Prince, Snakes, shapes, and gradient vector flow, IEEE Trans. Imag. Process. Vol 7, No. 3, pp.61-79, Feb. 1997

[12] J. LI, X. Yang, P F. Shi, A Fast Level Set Approach to Image Segmentation Based on Mumford-Shah Model[J], Chinese Computers, 2002, 25(1):1176-1183. (In Chinese) 